\title{
Magneto-optical properties and recombination dynamics of isoelectronic bound excitons in $\mathrm{ZnO}$
}

S. L. Chen, Weimin Chen and Irina Buyanova

\author{
Linköping University Post Print
}

Tweet

N.B.: When citing this work, cite the original article.

Original Publication:

S. L. Chen, Weimin Chen and Irina Buyanova, Magneto-optical properties and recombination dynamics of isoelectronic bound excitons in $\mathrm{ZnO}, 2014$, AIP Conference Proceedings, (1583), 186.

http://dx.doi.org/10.1063/1.4865632

Copyright: American Institute of Physics (AIP) http://www.aip.org/

Postprint available at: Linköping University Electronic Press

http://urn.kb.se/resolve?urn=urn:nbn:se:liu:diva-93892 


\section{AIP $\mid$ proceedings}

\section{Magneto-optical properties and recombination dynamics of isoelectronic bound excitons in $\mathrm{ZnO}$}

S. L. Chen, W. M. Chen, and I. A. Buyanova

Citation: AIP Conference Proceedings 1583, 186 (2014); doi: 10.1063/1.4865632

View online: http://dx.doi.org/10.1063/1.4865632

View Table of Contents: http://scitation.aip.org/content/aip/proceeding/aipcp/1583?ver=pdfcov

Published by the AIP Publishing

\section{Articles you may be interested in}

Recombination dynamics of a localized exciton bound at basal stacking faults within the m-plane ZnO film Appl. Phys. Lett. 105, 011106 (2014); 10.1063/1.4887280

Exciton recombination dynamics in single $\mathrm{ZnO}$ tetrapods

AIP Conf. Proc. 1566, 77 (2013); 10.1063/1.4848293

Dynamics of donor bound excitons in $\mathrm{ZnO}$

Appl. Phys. Lett. 102, 121103 (2013); 10.1063/1.4798531

Magneto-optical spectrum of $\mathrm{ZnO}$ nanorods

J. Appl. Phys. 111, 044305 (2012); 10.1063/1.3686139

Optical properties of the isoelectronic trap $\mathrm{Hg}$ in $\mathrm{ZnO}$

Appl. Phys. Lett. 82, 3448 (2003); 10.1063/1.1576912 


\title{
Magneto-Optical Properties and Recombination Dynamics of Isoelectronic Bound Excitons in ZnO
}

\author{
S. L. Chen, W. M. Chen, and I. A. Buyanova \\ Department of Physics, Chemistry and Biology, Linköping University, 58183 Linköping, Sweden.
}

\begin{abstract}
Magneto-optical and time-resolved photoluminescence (PL) spectroscopies are employed to evaluate electronic structure of a bound exciton (BX) responsible for the $3.364 \mathrm{eV}$ line (labeled as $\mathrm{I}^{*}{ }_{1}$ ) in bulk $\mathrm{ZnO}$. From timeresolved PL spectroscopy, $\mathrm{I}_{1}^{*}$ is concluded to originate from the exciton ground state. Based on performed magneto-PL studies, the g-factors of the involved electron and hole are determined as being $\mathrm{g}_{\mathrm{e}}=1.98$ and $\mathrm{g}_{\mathrm{h}}{ }^{\prime \prime}\left(\mathrm{g}_{\mathrm{h}}^{\perp}\right)=1.2(1.62)$, respectively. These values are nearly identical to the reported g-factors for the $\mathrm{I}^{*}$ line in ZnO (Phys. Rev. B 86, 235205 (2012)), which proves that $\mathrm{I}_{1}^{*}$ should have a similar origin as $\mathrm{I}^{*}$ and should arise from an exciton bound to an isoelectronic center with a hole-attractive potential.
\end{abstract}

Keywords: $\mathrm{ZnO}$, isoelectronic center, bound exciton. PACS: 78.55.Et, 71.35.Ji, 78.20.Ls, 71.55.Gs.

\section{INTRODUCTION}

$\mathrm{ZnO}$ is a direct wide bandgap semiconductor that continues to attract extensive research interest. A large binding energy for free excitons (FXs) in this material makes possible highly efficient FX radiative recombination at room temperature, advantageous for the applications of $\mathrm{ZnO}$ as an efficient ultra-violet light emitter and also for solidstate white lighting. At low temperatures, FXs become trapped by various unintentional impurities forming bound excitons (BXs) that give rise to a rich variety of sharp photoluminescence (PL) lines within the near-band-edge spectral range [1]. Most of these BX lines were identified as excitons bound to either neutral or ionized shallow donors, i.e. $\mathrm{D}^{0} \mathrm{Xs}$ or $\mathrm{D}^{+} \mathrm{Xs}$, respectively $[1,2]$. The charge state of the involved donors could be determined from magneto-PL measurements performed in an external magnetic field $\mathrm{B}$ applied perpendicular to the c-axis of $\mathrm{ZnO}$.

Besides the DXs discussed above, several BX lines of a different origin have recently been reported in $\mathrm{ZnO}[3$, 4]. Based on detailed magneto-optical and transient PL studies we have showed [4] that one of these lines, i.e. the I* line at $3.3621 \mathrm{eV}$, arises from an exciton bound to an isoelectronic center with a hole-attractive potential which partially quenches the orbital angular momentum of the bound hole. In this paper, we extend these studies to another BX from this series, namely $\mathrm{I}_{1}{ }_{1}$ at $3.364 \mathrm{eV}$, and show that it has the same electronic structure as $\mathrm{I}^{*}$. It is also found that the intensity of $\mathrm{I}^{*}\left(\mathrm{I}_{1}^{*}\right)$ correlates with the intensity of the so-called $\mathrm{I}_{6}\left(\mathrm{I}_{4}\right)$ line due to $\mathrm{Al}(\mathrm{H})$ impurity [1]. This may suggest that the isoelectronic center responsible for the $\mathrm{I}^{*}\left(\mathrm{I}_{1}{ }_{1}\right)$ transition contains an $\mathrm{Al}(\mathrm{H})$ atom as a part of the center.

\section{SAMPLES AND METHODS}

The investigated samples were commercially available c-plane bulk $\mathrm{ZnO}$ single crystals from different suppliers including Eagle Picher Co., Tokyo Denpa Co. and Cermet Inc. Magneto-PL measurements were carried out at 2K in a split-coil superconducting magnet. A pulsed Ti: sapphire laser with a repetition rate of $76 \mathrm{MHz}$ was used as an excitation source during time-resolved PL measurement. The transient PL was detected by a streak camera system combine with a $0.5 \mathrm{~m}$ single grating monochromator. The continuous-wave PL was excited by a solid state laser emitting at $266 \mathrm{~nm}$ and was detected by a photomultiplier tube (PMT) combined with a $0.8 \mathrm{~m}$ double-grating monochromator.

\section{EXPERIMENTAL RESULTS AND DISCUSSION}

Figure 1(a) presents typical PL spectra measured at 2K from Ealge Picher (the solid line) and Cermet (the dashed line) $\mathrm{ZnO}$. Within the near-band-edge spectral range both spectra contain a rich pattern of lines due to DX

International Conference on Defects in Semiconductors 2013

AIP Conf. Proc. 1583, 186-189 (2014); doi: 10.1063/1.4865632

(C) 2014 AIP Publishing LLC 978-0-7354-1215-6/\$30.00 
transitions. The intense lines labeled as $\mathrm{I}_{4}, \mathrm{I}_{6}, \mathrm{I}_{7}$ and $\mathrm{I}_{8}$ originate from $\mathrm{D}^{0} \mathrm{Xs}$ where the involved hole belongs to the top-most $\mathrm{A}$-valence band (VB), i.e. $\mathrm{D}^{0} \mathrm{X}^{\mathrm{A}}$. Lying about $4.4 \mathrm{meV}$ above these lines are the weaker and spectrally broader peaks related to the $\mathrm{D}^{0} \mathrm{X}$ transitions involving a $\mathrm{B}-\mathrm{VB}$ hole $[5,6], \mathrm{D}^{0} \mathrm{X}^{\mathrm{B}}$ - see e.g. the $\mathrm{I}_{6}^{\mathrm{B}}$ line in Fig. 1(a). In addition, the spectra contain emission lines at $3.3621 \mathrm{eV}$ and $3.3639 \mathrm{eV}$ which are labeled as $\mathrm{I}^{*}$ and $\mathrm{I}_{1}^{*}$, respectively. We note that the $\mathrm{I}^{*}$ intensity is the highest in Cermet $\mathrm{ZnO}$, where the PL spectra are dominated by the $\mathrm{I}_{6}$ emission

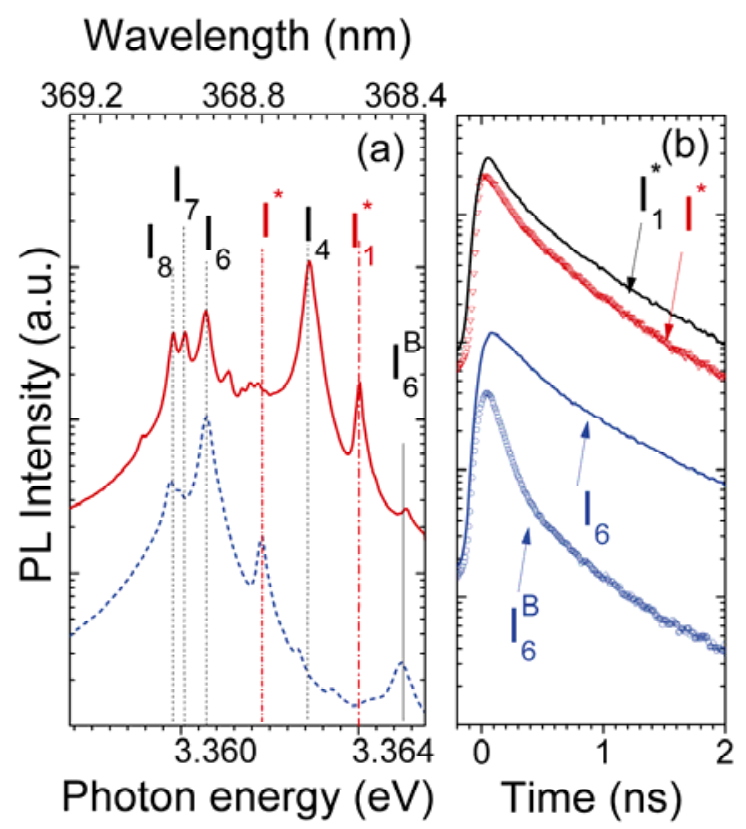

FIGURE 1. (Color online) (a) Photoluminescence spectra measured at $2 \mathrm{~K}$ from the Cermet (the dotted line, blue online) and Eagle Picher (the solid line, red online) ZnO. (b) PL decays measured at $2 \mathrm{~K}$ for the specified excitonic emissions. For clarity, all decay curves are offset vertically.

due to $\mathrm{DX}$ bound to an $\mathrm{Al}$ donor [1]. On the other hand $\mathrm{I}_{1}^{*}$ is the most pronounced line in the samples where $\mathrm{I}_{4}$ dominates.

The observed correlation may suggest that $\mathrm{I}^{*}$ and $\mathrm{I}_{1}^{*}$ stem from the excited state of $\mathrm{I}_{6}$ and $\mathrm{I}_{4}$, respectively, e.g. from an exited vibrational-rotational state. However, the transient dynamics of $I^{*}$ from our recent time-resolved PL study [4] suggests that this transition originates from the exciton ground state, as the $\mathrm{I}^{*}$ decay was found to be even longer than that of the $\mathrm{I}_{6}$ transition. To find out whether $\mathrm{I}_{1}^{*}$ is associated with an excited state or the ground state, we performed time-resolved PL measurement. The results of these measurements are shown in Fig.1b. For comparison, PL decays of $\mathrm{I}_{6}, \mathrm{I}_{6}^{\mathrm{B}}$, and $\mathrm{I}^{*}$ transitions are also shown. In $\mathrm{ZnO}$, PL decays of excitonic transitions that stem from the exciton ground state (such as $\mathrm{I}_{6}$ and $\mathrm{I}^{*}$ ) are relatively slow, i.e. of the order of 0.7-1.1 ns that is determined by their recombination lifetimes [7]. On the other hand, PL transients of excitonic transitions that originate from exciton excited states are much faster due to fast energy relaxation from the excited to the ground state [6]. This fast transient component is obvious, e.g. in the $\mathrm{I}_{6}{ }^{\mathrm{B}}$ decay - see Fig.1(b). As is obvious from Fig. 1(b), the fast decay component is not present in the $\mathrm{I}^{*}$ decay, which is in fact very similar to that of the $\mathrm{I}_{6}$ and $\mathrm{I}^{*}$ transitions. This provides the evidence that the $\mathrm{I}_{1}^{*}$ transition originates from the BX ground state, similar to $\mathrm{I}^{*}$.

Let us now discuss magneto-optical properties of $I^{*}{ }_{1}$. Figure 2 displays magneto-PL spectra measured within the spectral range of the $\mathrm{I}_{4}$ and $\mathrm{I}_{1}^{*}$ transitions from the Eagle Picher ZnO. In the Faraday geometry $(\mathbf{B}\|\mathbf{c}, \mathbf{k}\| \mathbf{c}), \mathrm{I}^{*}{ }_{1}$ linearly splits into two Zeeman components which have $\sigma^{+}$and $\sigma^{-}$polarization, as shown by the solid and dotted lines in Figure 2(a). 


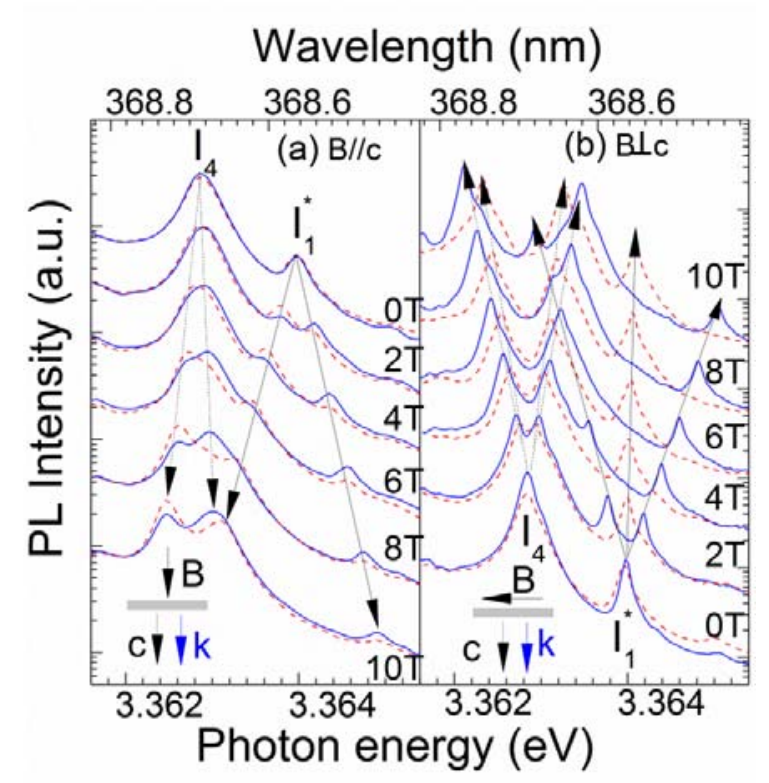

FIGURE 2. (Color online) (a) Magneto-PL spectra of the $\mathrm{I}_{4}$ and $\mathrm{I}_{1}{ }_{1}$ transitions measured in the Faraday geometry $(\mathbf{B} \| \mathbf{c}) . \sigma^{+}$and $\sigma^{-}$polarized emissions are represented by the solid (blue online) and dashed (red online) lines, respectively. (b) Magneto-PL spectra of the $\mathrm{I}_{1}{ }_{1}$ line measured in the Voigt geometry $(\mathbf{B} \perp \mathbf{c})$. the PL emissions are detected in the $\mathbf{E} \perp \mathbf{B}$ (the solid curves, blue online) and $\mathbf{E} \| \mathbf{B}$ (the dashed curves, red online) polarizations. The spectra measured at different fields are vertically shifted, for clarity.

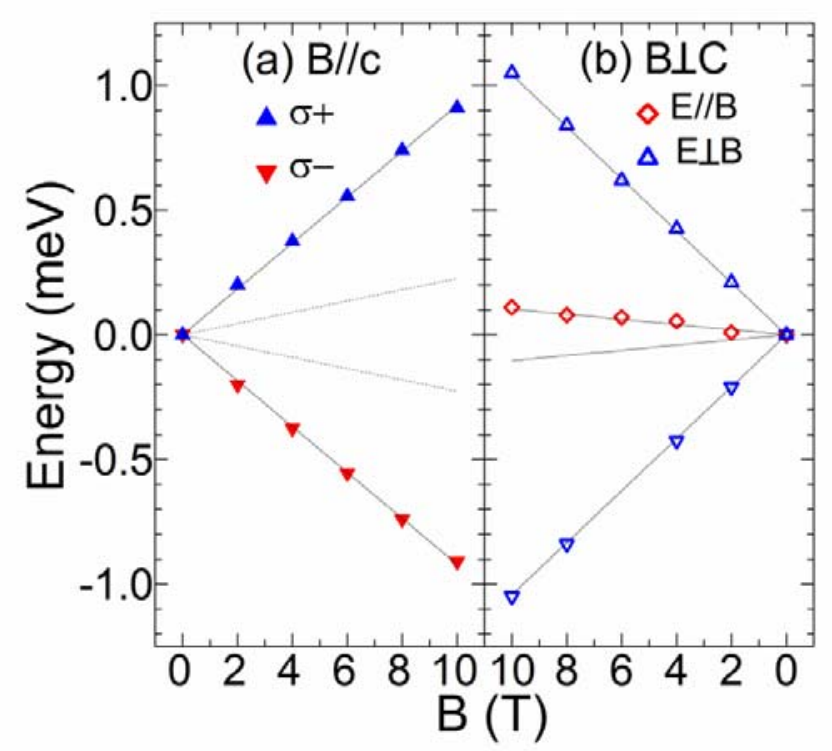

FIGURE 3. (Color online) Zeeman splitting of the $\mathrm{I}_{1}^{*}$ line as a function of a magnetic field in (a) Faraday and (b) Voigt configurations. The energy positions of all Zeeman peaks are plotted relative to their center-of-gravity. Symbols represent the experimental data, while the lines are fitting curves which yield the following electron and hole g-factors: $\mathrm{g}_{\mathrm{e}}=1.98, \mathrm{~g}_{h}^{\prime \prime}=1.2$, and $g_{h}^{\perp}=1.62$. The solid and dashed lines represent allowed and forbidden transitions, respectively. 
When the magnetic field B is directed perpendicular to the c-axis, i.e. in the Voigt geometry $(\mathbf{B} \perp \mathbf{c}, \mathbf{k} \| \mathbf{c})$, an additional Zeeman peak appears. Its energy position shifts to higher energies with increasing B. All Zeeman components in the Voigt geometry become linearly polarized with the outer and inner pair emitting in the $(\mathbf{E} \perp \mathbf{B}$, $\mathbf{E} \perp \mathbf{c})$ and $(\mathbf{E} / \mathbf{B}, \mathbf{E} \perp \mathbf{c})$ polarizations, respectively (see Fig. 2(b)). Here $\mathbf{E}$ denotes the electric field vector of the light emission. The energy positions of all Zeeman components of the $\mathrm{I}_{1}{ }_{1}$ line are plotted as a fan diagram in Figure 3 . The displayed Zeeman splittings are calculated relative to the center-of-gravity of $\mathrm{I}_{1}^{*}$, to compensate for a small diamagnetic shift. By fitting the Zeeman splitting of the outer components in different geometries, effective exciton g-factors could be derived as $g_{e x} \|=3.18$ and $g_{\text {exc }} \perp=3.60$. It should be noted that the deduced excitonic g-factors are much larger than the known values for $\mathrm{D}^{0} \mathrm{X}$ (e.g. the $\mathrm{I}_{4}$ line in Fig. 2) and $\mathrm{D}^{+} \mathrm{X}$ [1]. On the other hand, the Zeeman pattern and g-factors of $\mathrm{I}_{1}^{*}$ are consistent with the previously reported values for the $\mathrm{I}^{*}$ line $\left(\mathrm{g}_{\mathrm{ex}}{ }^{\|}=3.25\right.$ gexc $\left.{ }^{\perp}=3.62\right)$ [4].

The similarity between the transient and magneto-optical properties of $\mathrm{I}_{1}{ }_{1}$ and $\mathrm{I}^{*}$ suggests a similar origin for both transitions. This in turn implies that the $\mathrm{I}_{1}^{*}$ line arises from an exciton bound to an isoelectronic center. Following the analysis presented in [4], the electron and hole g-factors for $\mathrm{I}_{1}^{*}$ could be determined as $\mathrm{g}_{\mathrm{e}}=1.98$ and $g_{h}^{\prime \prime}\left(g_{h}^{\perp}\right)=$ 1.2 (1.62), respectively. The deduced value of the electron g-factor is almost identical to the conduction band electron g-value. On the other hand, the hole g-values significantly deviate from the known values of $g_{h}^{\prime \prime}=-(1.0-1.3)$ and $g_{h}^{\perp}=0.1-0.3$ for effective-mass holes in $\mathrm{D}^{0} \mathrm{X}^{\mathrm{A}}[1-3]$. This mismatch of the hole g-factors evidences that the hole should be tightly bound at the involved isoelectronic center. In other words, the center has a hole-attractive potential and, therefore, traps the hole as a primary bound particle. This is followed by binding of an electron due to Coulomb-like potential. As the screened residual contribution from the defect core potential is small, the electron in the BX complex retains the effective-mass character reflected by its binding energy and g-factor.

\section{CONCLUSIONS}

In conclusion, based on comprehensive magneto-optical and time-resolved PL measurements we have shown that both PL dynamics and Zeeman properties of the $\mathrm{I}_{1}^{*}$ transition are very similar to those reported most recently for the $\mathrm{I}^{*}$ line. We, therefore, suggest that both BXs have the same electronic structure and arise from excitonic transitions at isoelectronic centers with a hole-attractive potential. The electron and hole g-factors of the $\mathrm{I}_{1}^{*}$ exciton are derived as $\mathrm{g}_{\mathrm{e}}=1.98$ and $g_{h}^{\prime \prime}\left(g_{h}^{\perp}\right)=1.2(1.62)$. It is also found that the intensities of the $\mathrm{I}_{1}^{*}$ and $\mathrm{I}^{*}$ lines correlate with the intensities of the $\mathrm{I}_{4}$ and $\mathrm{I}_{6}$ transitions, respectively. This suggests that the isolectronic center responsible for the $\mathrm{I}_{1}{ }_{1}$ may involve an $\mathrm{H}$ atom, whereas an $\mathrm{Al}$ atom may be a part of the isolectronic center giving rise to the $\mathrm{I}^{*}$ transition.

\section{REFERENCES}

1. B. K. Meyer, H. Alves, D. M. Hofmann, W. Kriegseis, D. Forster, F. Bertram, J. Christen, A. Hoffmann, M. Straßburg, M. Dworzak, U. Haboeck, A. V. Rodina, Phys. Status Solidi B 241, 231-260 (2004).

2. A. V. Rodina, M. Strassburg, M. Dworzak, U. Haboeck, A. Hoffmann, A. Zeuner, H. R. Alves, D. M. Hofmann, and B. K. Meyer, Phys. Rev. B 69, 125206/1-9 (2004).

3. L. Ding, B. K. Li, H. T. He, W. K. Ge, J. N. Wang, J. Q. Ning, X. M. Dai, C. C. Ling, and S. J. Xu, J. Appl. Phys. 105, 053511/1-4 (2009)

4. S. L. Chen, W. M. Chen, and I. A. Buyanova, Phys. Rev. B 86, 235205/1-7 (2012)

5. B. K. Meyer, J. Sann, S. Eisermann, S. Lautenschlaeger, M. R. Wagner, M. Kaiser, G.Callsen, J. S. Reparaz, and A. Hoffmann, Phys. Rev. B 82, 115207/1-8 (2010).

6. S. L. Chen, W. M. Chen, and I. A. Buyanova, Appl. Phys. Lett. 99, 091909/1-3 (2011).

7. S. L. Chen, W. M. Chen, and I. A. Buyanova, Appl. Phys. Lett. 102, 121103/1-4 (2013) 